\title{
Lateral Pillar is the Key in Supporting Pre-collapse Osteonecrosis of the Femoral Head: A Finite Element Model Analysis of Propensity-Score Matched Cohorts
}

\section{Ji Hoon Bahk}

Catholic University of Korea College of Medicine: Catholic University of Korea School of Medicine Woo-Lam Jo

Catholic University of Korea College of Medicine: Catholic University of Korea School of Medicine Seung-Chan Kim

Catholic University of Korea College of Medicine: Catholic University of Korea School of Medicine Soon-Yong Kwon

Catholic University of Korea College of Medicine: Catholic University of Korea School of Medicine Youngwook Lim ( $\nabla$ cmcoslpart2@gmail.com )

Catholic University of Korea https://orcid.org/0000-0001-5648-9755

\section{Research Article}

Keywords: Finite element analysis, osteonecrosis of the femoral head, lateral pillar, femoral head collapse, propensity-matched score

Posted Date: November 5th, 2021

DOI: https://doi.org/10.21203/rs.3.rs-1020678/v1

License: (c) (i) This work is licensed under a Creative Commons Attribution 4.0 International License. Read Full License

Version of Record: A version of this preprint was published at Journal of Orthopaedic Surgery and Research on December 1st, 2021. See the published version at https://doi.org/10.1186/s13018-02102875-8. 


\section{Abstract \\ Background}

This study was designed as a cohort study using propensity-score matching to age and gender for finite element model (FEM) analysis from pre-collapse CT images of collapsed and non-collapse hips. Through FEM analysis, a global graphical output around the hip joint can provide simple impression of stress distribution: concentration or dispersion.

\section{Methods}

32 hips with ARCO stage 2 or 3 ONFH who were on follow up for over one-year period were retrospectively reviewed. 16 hips with no interval progression of collapse were set as the study group, then 16 hips with progression of collapse which required arthroplasty were set as the control group using propensity-score matching. FEM was generated through Mechanical Finder for each patient, then 4500 Newtons of load was applied to $1,000 \mathrm{~mm}^{2}$ area at the top of iliac crest to analyze equivalents for yield stress.

\section{Results}

Age, sex, and BMI had no significant differences, while location $(p=0.015)$ was lateral, and size $(p=0.015)$ was significantly greater in the collapsed group. Non-collapsed hips mostly exhibited stress dispersion allocated to medial and lateral pillars, while collapsed hips exhibited stress concentration focused on the lateral pillar and the primary compression trabecula. $(p=0.001)$

\section{Conclusion}

FEM analysis suggests stress concentration to the lateral pillar and the primary compression trabeculae can predict future collapse in ONFH with high probability. Results provide a simple and intuitive, yet valuable information to aid surgeons. Therefore, especially for young patients, holding out the lateral pillar through joint preserving procedures might be the key in preventing further collapse.

\section{Background}

The natural history of osteonecrosis of the femoral heads (ONFH) is still an area of uncertainty, since some patients with early stages of ONFH might undergo collapse in time while others do not exhibit any progression throughout follow up. Therefore, the matter of utmost concern to surgeons has been the prediction of collapse progression of the femoral head.[1] In an effort, various staging systems based on necrotic size, location, and presence of subchondral fractures or collapse have been widely used in practice. Among them, Ficat and Alert classification[2], University of Pennsylvania system[3], the Japanese Investigation Committee (JIC) classification[4], and ARCO (Association Research Circulation 
Osseous) international classification of osteonecrosis have been widely used in order to make the ultimate treatment choice: surgery or observation. Subsequently, Kerboul combined index was proposed to predict collapse using sum of necrotic angles in MRI images[5] and another latest study suggested maximum area in coronal position (MAC) of initial bone resorption to be an indicator of collapse.[6]

More recently, since the introduction and application of finite element model (FEM) analysis on ONFH, novel studies have been reported in advanced attempt to reveal better indicators.[7] Through FEM study, Utsunomiya et al. concluded that lateral boundaries of the necrotic lesion lead to subchondral fractures and collapse.[8] Additionally, in FEM interpretation via peak von Mises stress, Wen et al. emphasized the significance of the lateral pillar in progression of the disease[9, 10], and Li et al. suggested that maximal level of stress on weight-bearing surfaces as a new biomechanical marker for the prediction.[11]

We designed a retrospective cohort study using propensity-score matching to age, gender, and BMI for FEM analysis from pre-collapse CT scan images of collapsed and non-collapse hips. ARCO staging, size, and location were primarily compared between the two cohorts. Furthermore, FEM was generated to determine how the stress distribution is taking place at the femoral head, especially in the lateral pillar and the primary compression trabeculae. Through FEM analysis, rather than locally focusing on quantification, a global graphical output around the hip joint was obtained which gives us a simple impression of the stress distribution: concentration or dispersion.

\section{Methods}

This study was approved prior to initiation by the Institutional Review Board. Informed consent was waived by the board. In total, 32 hips in 32 distinct patients with ARCO stage 2 or 3 ONFH which was diagnosed with both plain radiographs and MRI who were on follow up for over than one-year period between January 2016 and December 2018 were retrospectively enrolled in this study. 16 hips with no interval progression of collapse were set in the study group (group A), then in turn, 16 hips with progression of collapse which eventually required arthroplasty were set as the control group (group B) by using propensity-score matching to age, sex, and body mass index (BMI).

\section{Finite Element Model generation}

For analysis, three-dimensional FEM of each patient were generated using Mechanical Finder version 10.0 (Research Center for Computational Mechanics, Tokyo, Japan) and digital imaging and communication in medicine (DICOM) images obtained from routine initial pelvic bone CT (120 kVp, 1.0mm slice thickness, SOMATOM Definition, Siemens Medical Solutions, Forchheim, Germany). Extracted DICOM images were imported into Mechanical Finder, then ranges of interest (ROI) were initially extracted using computational methods to firstly select coarse areas over arbitrary Hounsfield unit thresholds. Then additionally, fine ROI boundaries were selected by meticulous manual identification to reflect more accurate necrotic areas to the FEM. Subsequently, ANSYS version 19.2 (ANSYS, Inc., Canonsburg, Pennsylvania, USA) was loaded to Mechanical Finder for mesh generation. 
For outer surface of the cortical bone, $0.5 \mathrm{~mm}$ thickness iso-surface external mesh was automatically generated where for the trabecular bone, internal mesh was generated using $1 \mathrm{~mm}$-sized tetrahedral elements. Then stepwise material property was configured with inhomogeneous bone material settings using Keyak (1998) preset for Young's Modulus and for yield stress. Additionally, Poisson's Ratio was set to 0.4 and distribution of Drucker-Prager was utilized for yield criterion. After completion of FEM generation, stress load of 4,500 Newtons $(\mathrm{N})$ was added to manually pointed $1,000 \mathrm{~mm}^{2}$ area at the top of ipsilateral iliac crest, with the vector parallel to the vertical axis of the body. (Fig. 1) Ultimately, final analysis was performed to yield qualitative and quantitative results of the stress distributions around the hip joint, which includes three-dimensional output figures. (Fig. 2)

\section{Study outcomes}

Changes in necrotic areas were reassessed using CT images at follow up and was again classified using ARCO international classification of osteonecrosis. Additionally, the location of center of necrosis was assessed in accordance to trisections of femoral head on coronal plane[12]: medial, central, or lateral. The size of necrosis was measured by the percent area (extent) of the necrotic portion using proportional expression using the longest mediolateral and anteroposterior length of necrotic lesion and the largest mediolateral and anteroposterior diameter of the femoral head. [13]

Primary outcome was set as equivalents for yield stress (\%) which signifies the compression force applied around the hip joint of concern. The ultimate outcome of the FEM was provided in the visual form of figures which quantitatively displays the coronal cross sections of the joint, hence intuitively showing either 'stress concentration' or 'stress dispersion' is being engaged to the proximal femur. The results were classified as stress dispersion (Fig. 3a) when no focal concentration of stress was observed, and as stress concentration (Fig. 3c) when convergence of load transfer to the lateral pillar was observed. Intermediate results were assessed as stress dispersion in that significant focal concentration was not observed (Fig. 3b)

\section{Statistical analysis}

Suspected confounding variables of age, sex, and BMI were used for propensity score analysis between collapsed and non-collapsed groups. Paired t-test was used to confirm match design. Mann-Whitney tests were used for analysis of numerical variables including age, BMI, and necrotic size. In addition, Fisher's exact tests were used to compare gender, location of necrosis, and the final results of stress distribution between the two groups. All statistical analyses were performed through Statistical Package for the Social Sciences (Version 20.0; SPSS, Chicago, Illinois, USA) For propensity score matching, PS Matching R plugin (version 3.0) on R software was used. (Version 2.12.0, R Development Core Team, Vienna, Austria) Cutoff $p$-value of $<0.05$ was used to determine statistically significant results.

\section{Results}


Demographics of age ( $49.4 \pm 14.2$ vs. $48.7 \pm 13.5, p=0.958)$ and gender (male to female, 9:7 vs. 9:7, $p=1.000$ ) exhibited no significant difference between the two groups, reassuring propensity-scored matching. In addition, $\mathrm{BMI}$ also had no significant difference (23.3 \pm 4.7 vs. $25.1 \pm 6.2, p=0.944)$. (Table 1 ) Locations of the necrosis were all in the lateral trisection for group $A$, while group $B$ were distributed to central or medial $(n=8)$ and lateral $(n=8)$ trisections $(p=0.015)$. Size of necrosis was larger in group $B$ $(46.8 \pm 20.3 \%$ vs. $64.1 \pm 33.6 \%, p=0.015)$. (Table 2 ) Upon reassessment at over one-year follow up, patients in group $A$ had resulting $A R C O$ stage of $2 B(n=2), 2 C(n=10), 3 B(n=1)$, and $3 C(n=3)$, whereas all patients in group $B$ proceeded to group $3 C(n=16)$ who experienced progression of collapse.

Table 1

Demographics of 32 hips in 32 distinct patients with ONFH.

\begin{tabular}{|llll|}
\hline & Non-collapsed & Collapsed & $p$-value \\
\hline Age (years) & $49.4 \pm 14.2$ & $48.7 \pm 13.5$ & 0.958 \\
& $(21-75)$ & $(26-79)$ & \\
\hline Sex (male:female) & $9: 7$ & $9: 7$ & 1.000 \\
\hline BMl $\left(\mathrm{kg} / \mathrm{m}^{2}\right)$ & $23.3 \pm 4.7$ & $25.1 \pm 6.2$ & 0.944 \\
\hline
\end{tabular}

For the end result, among group A, most cases exhibited stress dispersion $(n=10)$ which was achieved by distribution of forces diverging to medial and lateral pillars (Fig. 3A), while a portion ( $n=3)$ had less discrete dispersal but still stress did not concentrate to the lateral pillar nor the primary compression trabeculae. (Fig. 3b) Few showed stress concentration through the lateral pillar $(n=3)$. Overall, stress dispersion mainly consisted of group A ( $\mathrm{n}=13(81.2 \%))$.

On the other hand, in group B, stress concentration was mostly observed ( $n=14(87.5 \%))$ where the stress is mainly delivered through the hip joint in a penetrating fashion, especially focusing on the lateral pillar of the femoral head, which then vertically converges along the primary compression trabecula of femoral neck. (Fig. 3c) Minority of group B exhibited stress dispersal ( $n=2)$, which still showed collapse progression and required total hip arthroplasty.

Altogether, stress dispersal was mostly observed in the non-collapsed group whereas stress concentration was dominantly observed in the collapsed group. $(p=0.001)$ (Table 2$)$ 
Table 2

Initial characteristics of osteonecrosis and FEM analysis results of stress distribution $\left({ }^{*} p<0.05\right)$

\begin{tabular}{|llll|}
\hline & Non-collapsed & Collapsed & $p$-value \\
\hline $\begin{array}{l}\text { Location } \\
\text { (central or medial : lateral) }\end{array}$ & $8: 8$ & $0: 16$ & $0.015^{\star}$ \\
\hline Size of necrosis (\%) & $46.9 \pm 20.3$ & $64.1 \pm 33.6$ & $0.015^{\star}$ \\
& $(16.7-85.8)$ & $(64.1-92.2)$ & \\
\hline Stress concentration & $3: 13$ & $14: 2$ & $0.001^{*}$ \\
(Yes : No) & & & \\
\hline
\end{tabular}

\section{Discussion}

The prediction of collapse progression has been the key question in treating ONFH patients. Evolvement of classification systems and techniques in interpreting imaging studies were directed to elucidate such inquiry. Thus, indications of each treatment options including conservative care, joint preserving techniques, or arthroplasties have been widely studied, with a goal of basic consensus to preserve one's natural hip joint as long as possible. In a recent study based on U.S. nationwide database, Sodhi et al. reported rates of arthroplasty (94.03\%) were far greater than those of other procedures including osteotomy, partial arthroplasty, core decompression, and bone graft.[14] However, in young population, joint preserving techniques should be considered in prior to arthroplasties due to the latter's higher complication rate, invasiveness, and implant life span which could lead to revision surgery.[4, 14, 15]

In particular, biomechanical significance of the lateral pillar had been suggested in preventing collapse,[9, $10,16]$ but to date, cohort studies of which were scarcely reported.[11] Thus we designed a study of propensity-score matched cohorts to further minimize selection bias prone in retrospective data analysis. Additionally, FEM analysis was selected to investigate the fact that not all osteonecrosis placed in the lateral trisection undergo collapse, presumably due to dynamic basis rather than its sole mechanical structures. Hence, emphasis of this study is put on the phenomenon that not the absolute force on the lateral pillar but the presence of stress concentration on the critical areas determines collapse progression. Also, in this setting, it looks impracticable to set an absolute cutoff value in predicting collapse, in that it may vary under various local and global conditions among different individuals' hip joints.

For demographics, age $(p=0.958)$, sex $(p=1.000)$, and as well as BMI $(p=0.944)$ between the two groups had no significant difference as propensity scoring was estimated for age, sex, and BMI to minimize confounders. For pre-analytic comparison of characteristics of necrotic areas, location was classified as 'lateral' or 'non-lateral', where the latter includes centrally or medially located lesion based on the 
significance of the lateral pillar. All necrotic lesions in the collapsed group had pre-collapse lesions in the lateral trisection $(n=16)$, while lesions in the non-collapsed group were located half in the lateral $(n=8)$ and the other half in the non-lateral $(n=8)$ trisections. As a result, location $(p=0.015)$ and size $(p=0.015)$ had significant difference, which follows the current understandings of intrinsic risk factors of necrosis progression.

Upon the hypothesis of stress concentration on the lateral pillar might accelerate collapse, FEM analysis was conducted. To reflect extreme forces that can be applied to the hip joint, arbitrary stress load of $4,500 \mathrm{~N}$ was set considering that peak $870 \%$ of body weight can be applied when stumbling in a $53 \mathrm{~kg}$ weighing individual. [17] As a result, stress concentration was focused on the lateral pillar in $87.5 \%$ of hips in the collapsed group while stress dispersion through the pillars were observed in $81.2 \%$ of the hips in the non-collapsed group ( $p=0.001)$. Additionally, when stress concentration to the lateral pillar was present, distal force transmission through the primary compression trabeculae were always coupled. That is, stress concentration converging to the vertical axis would predict near-future collapse with high probability, whereas stress dispersion through medial (inferior) and lateral (superior) cortices of proximal femur is crucial in maintaining support of the anatomical structure. Therefore, the importance of structural support is emphasized not only at the lateral pillar, but inevitably also the primary compression trabeculae owing to its extended transmission of the yield stress distally.

Limitations of this study includes small sample size of 16 patients per cohort, hence propensity-score matching was used to reinforce clinical significance given the small size in this retrospective case cohort study. Second, this study lacks quantitative analysis via such as von Mises stress[9, 11], stress index[7], or value of equivalent stress.[8] But as stated above, emphasis of this study is put on the qualitative cognition on the stress distribution rather than suggesting quantified cutoff values. In turn, proving effects of reinforced support to the lateral pillars using buttresses such as with fibular strut grafts or tantalum rod implantation would be analyzed through FEM in the future studies.

In conclusion, FEM analyses of followed-up ONFH suggest stress concentration to the lateral pillar and the primary compression trabeculae predicts collapse with high probability. Graphical output as an end result in pre-collapse ONFH provides a simple and intuitive, but yet valuable information to aid surgeons in treatment selection. Moreover, FEM generation is achieved easily using ordinary CT data of a patient, depicting stress distribution which can be recognized at a glance. Therefore, especially for young patients, holding out the lateral pillar and the primary compression trabeculae through joint preserving procedures might be the key in preventing further collapse of the femoral head.

\section{Abbreviations}

ARCO: Association Research Circulation Osseous

BMI: body mass index

CT: computed tomography 
DICOM: digital imaging and communication in medicine

FEM: finite element model

MRI: magnetic resonance imaging

$\mathrm{N}$ : Newtons

ONFH: osteonecrosis of the femoral head

SPSS: Statistical Package for the Social Sciences

\section{Declarations}

\section{CONFLICT OF INTEREST}

The authors declare that they have no known competing financial interests or personal relationships that could have appeared to influence the work reported in this paper.

\section{ETHICS APPROVAL AND CONSENT TO PARTICIPATE}

This study was performed in line with the principles of the Declaration of Helsinki. Approval was granted and informed consent was waived by the Institutional Review Board of Seoul St. Mary's Hospital. (November 9, 2020, No. KC20RISI0276)

\section{CONSENT FOR PUBLICATION}

Not applicable.

\section{AVAILABILITY OF DATA AND MATERIAL (DATA TRANSPERANCY)}

The datasets used and analyzed during the current study are available from the corresponding author on reasonable request.

\section{COMPETING INTERESTS}

The authors declare that they have no known competing financial interests or personal relationships that could have appeared to influence the work reported in this paper.

\section{FUNDING STATEMENT}

This work was supported by the National Research Foundation of Korea (NRF) grant funded by the Korean government. (Ministry of Science and ICT) (Grant No. 2018R1C1B6002653). Additionally, it was supported by Research Fund of Seoul St. Mary's Hospital, The Catholic University of Korea. (Grant No. ZC21RISI0423) 


\section{AUTHOR'S CONTRIBUTIONS}

Young Wook Lim contributed to overall conceptualization, funding acquisition, supervised the study design and data curation, revised the manuscript, and acted as the corresponding author. Ji Hoon Bahk contributed as the first author in initial radiographic measurements, software utilization (FEM generation), visualization, and original manuscript writing. Woo-Lam Jo performed formal statistical analysis and also contributed in methodology and radiographic measurements. Seung-Chan Kim has contributed to radiographic data acquisition, formal analysis, and validation. Yong-Sik Kim contributed to conceptualization, project administration, and validation. All authors reviewed and approved the final manuscript.

\section{ACKNOWLEDGEMENT}

This work was supported by the National Research Foundation of Korea (NRF) grant funded by the Korean government. (Ministry of Science and ICT) (Grant No. 2018R1C1B6002653). Additionally, it was supported by Research Fund of Seoul St. Mary's Hospital, The Catholic University of Korea. (Grant No. ZC21RISI0423)

\section{SOFTWARE PROTOCOL}

Mechanical Finder version 10.0 (Research Center for Computational Mechanics, Tokyo, Japan)

RRID: SCR_021234

\section{References}

1. Kim S, Lim Y, Kwon S, Jo W, Heu J, Kim Y. Effect of leg-length discrepancy following total hip arthroplasty on collapse of the contralateral hip in bilateral non-traumatic osteonecrosis of the femoral head. The bone \& joint journal. 2019;101:303-10.

2. Ficat R. Idiopathic bone necrosis of the femoral head. Early diagnosis and treatment. The Journal of bone and joint surgery British volume. 1985;67:3-9.

3. Steinberg ME, Hayken GD, Steinberg DR. A quantitative system for staging avascular necrosis. The Journal of bone and joint surgery British volume. 1995;77:34-41.

4. Kuroda Y, Tanaka T, Miyagawa T, Kawai T, Goto K, Tanaka S, et al. Classification of osteonecrosis of the femoral head: Who should have surgery? Bone \& joint research. 2019;8:451-8.

5. Kerboul M, Thomine J, Postel M, d'Aubigne RM. The conservative surgical treatment of idiopathic aseptic necrosis of the femoral head. The Journal of bone and joint surgery British volume. 1974;56:291-6.

6. Shi S, Luo P, Sun L, Xie L, Yu T, Wang Z, et al. Prediction of the progression of femoral head collapse in ARCO stage 2-3A osteonecrosis based on the initial bone resorption lesion. The British Journal of Radiology. 2021;94:20200981. 
7. Yang J-W, Koo K-H, Lee M-C, Yang P, Noh MD, Kim S-Y, et al. Mechanics of femoral head osteonecrosis using three-dimensional finite element method. Archives of orthopaedic and trauma surgery. 2002;122:88-92.

8. Utsunomiya T, Motomura G, Ikemura S, Kubo Y, Sonoda K, Hatanaka H, et al. Effects of sclerotic changes on stress concentration in early-stage osteonecrosis: a patient-specific, 3D finite element analysis. Journal of Orthopaedic Research®. 2018;36:3169-77.

9. Wen P-F, Guo W-S, Zhang Q-D, Gao F-Q, Yue J-A, Liu Z-H, et al. Significance of lateral pillar in osteonecrosis of femoral head: a finite element analysis. Chinese medical journal. 2017;130:2569.

10. Wen P, Zhang Y, Hao L, Yue Ja, Wang J, Wang T, et al. The effect of the necrotic area on the biomechanics of the femoral head-a finite element study. BMC musculoskeletal disorders. 2020;21:1-8.

11. Li Tx, Huang Zq, Li Y, Xue Zp, Sun Jg, Gao Hh, et al. Prediction of Collapse Using Patient-Specific Finite Element Analysis of Osteonecrosis of the Femoral Head. Orthopaedic surgery. 2019;11:794800.

12. Hamada H, Takao M, Sakai T, Sugano N. Subchondral fracture begins from the bone resorption area in osteonecrosis of the femoral head: a micro-computerised tomography study. International orthopaedics. 2018;42:1479-84.

13. Nam KW, Kim YL, Yoo JJ, Koo K-H, Yoon KS, Kim HJ. Fate of untreated asymptomatic osteonecrosis of the femoral head. JBJS. 2008;90:477-84.

14. Sodhi N, Acuna A, Etcheson J, Mohamed N, Davila I, Ehiorobo JO, et al. Management of osteonecrosis of the femoral head: an up-to-date analysis of operative trends. The bone \& joint journal. 2020;102:122-8.

15. Hungerford DS. Osteonecrosis: avoiding total hip arthroplasty. The Journal of arthroplasty. 2002;17:121-4.

16. Sun W, Li Z-r, Wang B-L, Liu B-L, Zhang Q-D, Guo W-s. Relationship between preservation of the lateral pillar and collapse of the femoral head in patients with osteonecrosis. Orthopedics. 2014;37:e24-e8.

17. Bergmann G, Graichen F, Rohlmann A. Hip joint loading during walking and running, measured in two patients. Journal of biomechanics. 1993;26:969-90.

\section{Figures}




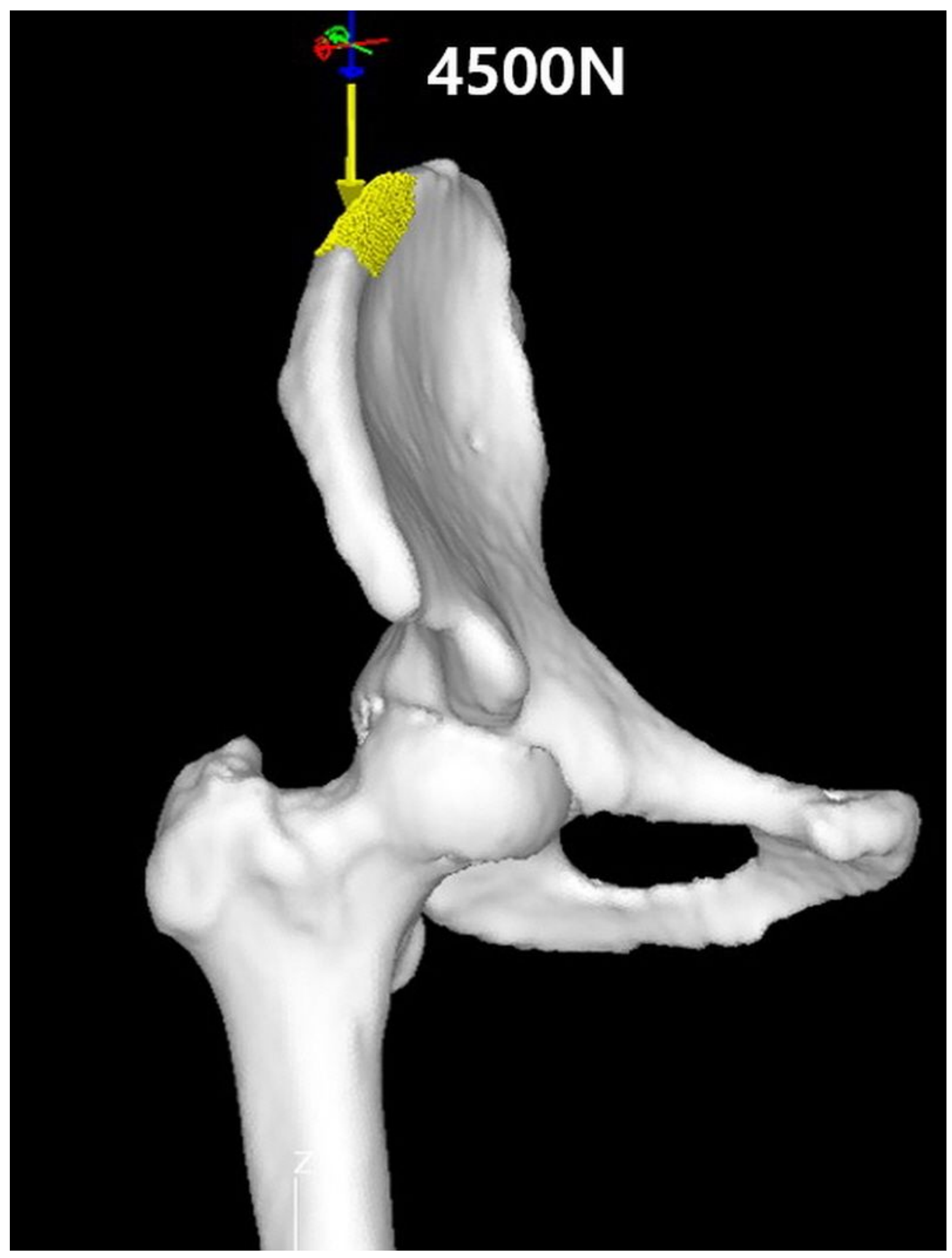

\section{Figure 1}

Virtual load application to FEM. Load of $4500 \mathrm{~N}$ based on - was applied to arbitrary 1,000mm2 area at the top of iliac crest with vector parallel to the vertical axis of body. (arrow) 


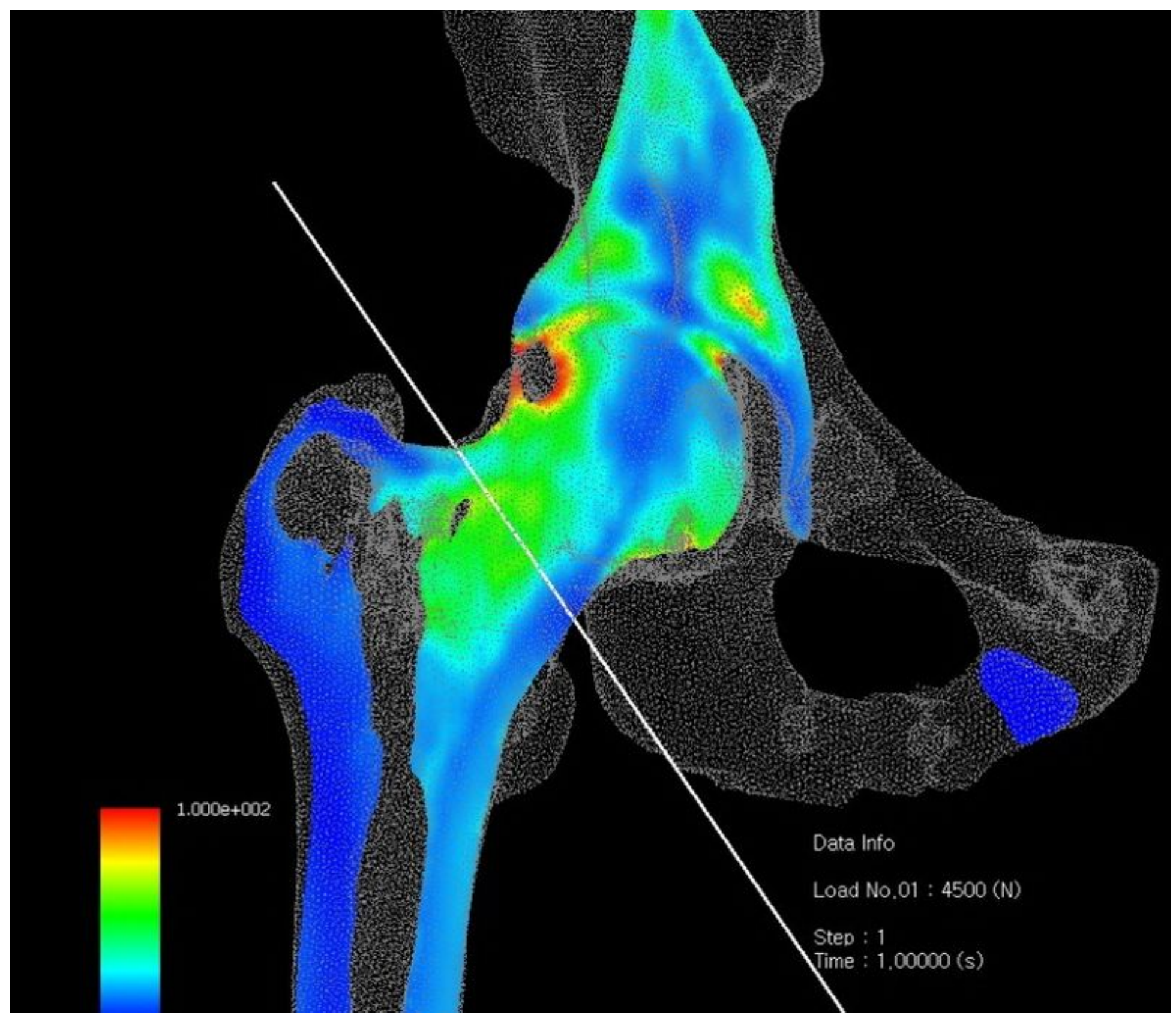

\section{Figure 2}

Final graphical output image after FEM analysis. Resulting stress distribution across the hip joint, through the proximal femur is visualized. Note an example from group A which has necrotic area at the lateral pillar, but stress dispersion prevents the femoral head from collapsing through time. 


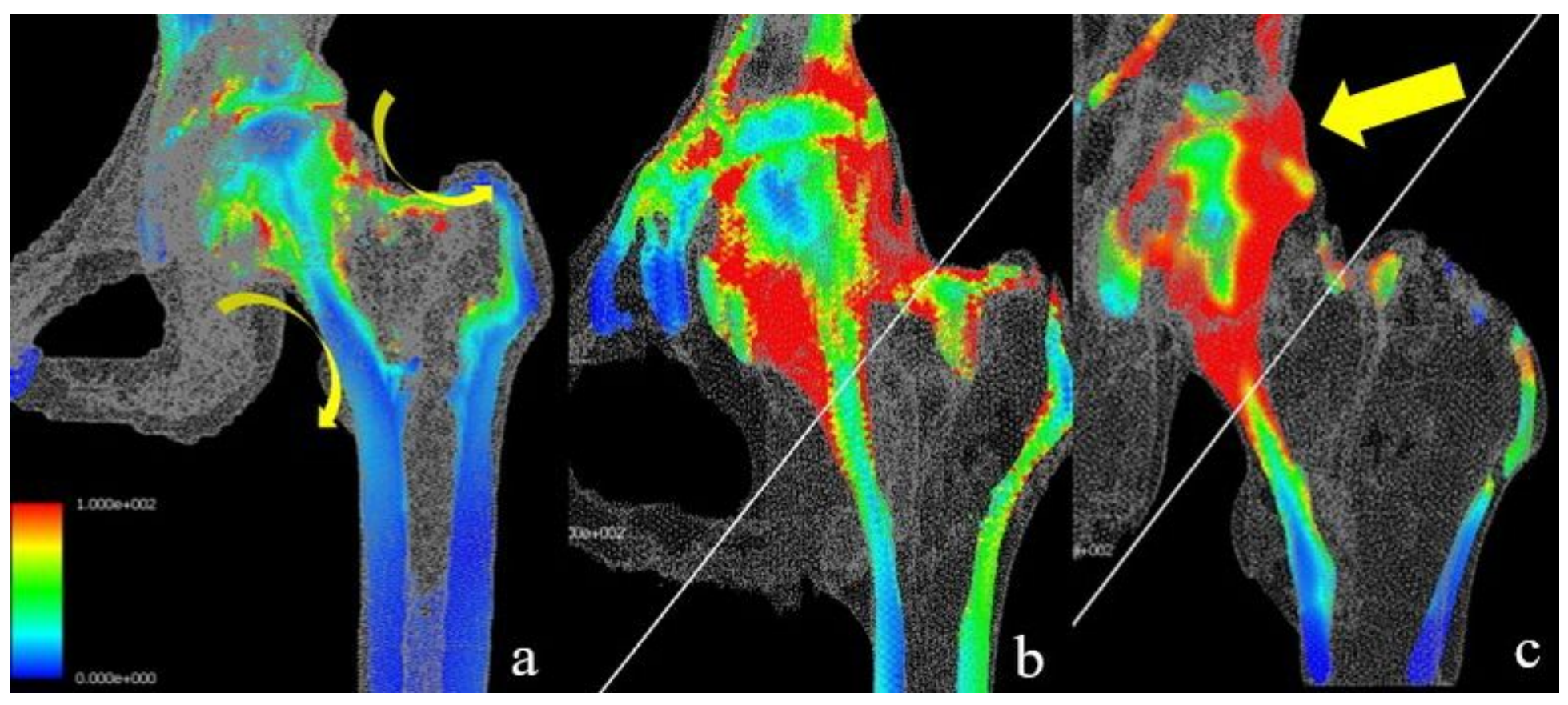

\section{Figure 3}

Representative final results of FEM analysis. Determining stress distribution conditions is focused on the if the concentration is taking place at the lateral pillar. Stress dispersion to the medial (inferior) and lateral (superior) cortices (curved arrows) is significantly dominant (81.2\%) in non-collapsed group (Fig. 3a). Likewise, intermediate results (Fig. 3b) were regarded as stress dispersion in the analysis. On the contrary, stress concentration to the lateral pillar (straight arrow) which proceeds to the primary compression trabeculae is markedly observed (87.5\%) in the collapsed group, which would predict collapse acceleration. (Fig. 3c) 